\title{
Letter
}

\section{A note on a mimetic scalar-tensor cosmological model}

\author{
Yevgeniya Rabochaya ${ }^{\mathrm{a}}$, Sergio Zerbini ${ }^{\mathrm{b}}$ \\ Dipartimento di Fisica, TIFPA-INFN, Università di Trento, Via Sommarive 14, 38123 Povo, Trento, Italy
}

Received: 18 September 2015 / Accepted: 1 February 2016 / Published online: 18 February 2016

(C) The Author(s) 2016. This article is published with open access at Springerlink.com

\begin{abstract}
A specific Hordenski scalar-gravity mimetic model is investigated within a FLWR space-time. The mimetic scalar field is implemented via a Lagrangian multiplier, and it is shown that the model has equations of motion formally similar to the original simpler mimetic matter model of Chamseddine-Mukhanov-Vikman. Several exact solutions describing inflation, bounces, and future-time singularities are presented and discussed.
\end{abstract}

\section{Introduction}

It is well known that General Relativity (GR) with additional suitable cosmological positive constant and Dark Matter, describes quite well a large part of the history of the Universe, including Dark Energy era. With an additional scalar degree of freedom, this model may also describe the primordial inflationary period, which is necessary for solving the horizon and flatness problem. This is essentially the socalled $\Lambda$-CDM model, or standard cosmological model, and it has been recently tested with high accuracy $[1,2]$.

For later comparison, we recall that in a flat FLRW spacetime

$\mathrm{d} s^{2}=-\mathrm{d} t^{2}+a(t)^{2} \delta_{i j} \mathrm{~d} x^{i} \mathrm{~d} x^{j}, \quad i, j=1,2,3$,

and GR plus ordinary matter with an equation of state $p=$ $\omega \rho, \rho$ density of radiation or matter, lead to the continuity equation

$\dot{\rho}+3 H(1+\omega) \rho=0$

and

$2 \dot{H}+3 H^{2}=-\omega \rho$,

where $H(t)$ is the usual Hubble parameter. When the solution of Eq. (2), namely $\rho(t)=\rho_{0} a(t)^{-3(1+\omega)}$, is taken into

\footnotetext{
a e-mail: rabochaya@ science.unitn.it

be-mail: zerbini@science.unitn.it
}

account, one has a non-linear second order differential equation for $a(t)$. Of course, in GR, one may make use of the Friedmann equation to arrive directly at the explicit solution for $a(t)$, a textbook result. In the presence of a scalar field, things are not so simple, and, in general, it is not easy to find exact solutions (see, for example $[3,4]$ ).

Furthermore, in the standard cosmological model, Dark Matter and Dark energy issues are still under investigation, since one has no clue what they are. In fact, it is well known that the dark energy effect is well parametrized by the inclusion of a tiny positive cosmological constant, but the coincidence and cosmological constant problems arise. In the physics of elementary particles there exist a few candidates for dark matter, but experimental verification is still lacking, and other alternatives are possible.

For these reasons, in this paper, we would like to consider a generalization of the so called mimetic dark matter-gravity models [5-7]. This proposal may be considered a minimal modification of GR, in which cosmological dark matter may be described. Soon it had also been realized [8,9] that this class of models are related to GR by singular disformal transformations. In particular the models [5-7] have been introduced by making use of a singular conformal transformation.

Disformal transformations were introduced by Bekenstein [10]. He was able to show that as a consequence of the diffeomorphism invariance of GR, any metric tensor $g_{\mu, v}$ may be parametrized by a fiducial matrix $l_{\mu, v}$ and by a scalar field $\phi$. As a consequence, $\phi$ seems to describe a new gravitational degree of freedom, but as soon as the disformal transformation is invertible, it turns out that no additional degrees of freedom are present, and GR is recovered. However, if the disformal transformation is singular, then the scalar $\phi$ becomes a new degree of freedom. This is a general fact [11]. We will not make use of this very powerful approach and we refer to the original papers. In fact, another equivalent approach is possible $[9,11,12]$, and within this approach a Lagrange multiplier is introduced $[13,14]$ in order to implement the "mimetic" constraint 
$\partial_{\mu} \phi \partial^{\mu} \phi=-1$.

Very recently, a general Horndeski scalar-tensor mimetic theory has been considered, and the two possible approaches, the singular disformal transformation and the Lagrange multiplier approach have been shown to be equivalent [11]. Concerning different aspects and generalization of mimetic gravity see, for example [15-20].

In this paper, in order to deal with the mimetic field $\phi$, we shall follow the Lagrange multiplier approach.

The outline of the paper is the following. In Sect. 2, the mimetic model is introduced. In Sect. 3 addresses a solution in the absence of matter, while in Sect. 4 matter or radiation is included. The paper ends with the conclusions and an Appendix.

We use units in which the reduced Planck mass is $M_{P}^{2}=1$.

\section{Mimetic scalar-tensor gravity model}

In this section we start with the following mimetic scalar tensor gravity model:

$I=\int_{\mathcal{M}} \mathrm{d}^{4} x \sqrt{-g}\left(\frac{R}{2}+\lambda\left(X-\frac{1}{2}\right)-V(\phi)\right)+I_{H}+I_{m}$

where $I_{m}$ is the usual matter-radiation action and the higher order contribution is given by

$$
\begin{aligned}
I_{H}= & \int_{\mathcal{M}} \mathrm{d}^{4} x \sqrt{-g}\left(\alpha\left(X R+\left(\nabla_{\mu} \nabla^{\mu} \phi\right)^{2}-\nabla_{\mu} \nabla_{\nu} \phi \nabla^{\mu} \nabla^{\nu} \phi\right)\right. \\
& \left.+\gamma \phi G_{\mu \nu} \nabla^{\mu} \nabla^{\nu} \phi-\beta \phi \nabla_{\mu} \nabla^{\mu} \phi\right)
\end{aligned}
$$

with $X=-\frac{1}{2} g^{\mu \nu} \partial_{\mu} \phi \partial_{\nu} \phi ; \lambda$ is a Lagrange multiplier and $\phi$ is the mimetic scalar field, and $\beta, \alpha$, and $\gamma$ are constants. The above Lagrangian is a particular case of the general Horndeski Lagrangian [21-23]. Some examples of Horndeski mimetic gravity models have been considered recently in [11].

When the constants $\beta, \alpha$, and $\gamma$ are vanishing, and in the absence of matter, the above model reduces to the original mimetic gravity proposed by Chamseddine and Mukhanov [5]. When $\alpha$ and $\beta$ are vanishing, the model reduces to the one studied in [24,25]; see also [20] for other aspects.

In order to study the dynamics of the above model in a flat FLRW space-time, one has to make use of

$\mathrm{d} s^{2}=-\mathrm{e}^{2 b(t)} \mathrm{d} t^{2}+a(t)^{2} \delta_{i j} \mathrm{~d} x^{i} \mathrm{~d} x^{j}, \quad i, j=1,2,3$,

here, for the sake of convenience, we write the lapse function as $\mathrm{e}^{b(t)}$, where $b(t)$ is an arbitrary dynamical variable which takes the value $b=0$ after variations. The action (5) may be written as a functional of $a(t), b(t)$, and $\lambda$. Variation with respect to $\lambda$ and assuming $\phi$ to depend only on $t$ give $\dot{\phi}^{2}=1$.

Thus, in the following, one may take $\phi=t$. Variation with respect to $b$ gives the generalized Friedmann equation and reads

$3 H^{2}(1-3 \alpha+3 \gamma)-V+\beta-\rho=\lambda$.

Here $H=\frac{\dot{a}}{a}$, the Hubble parameter, and $\rho$ is the matterradiation density.

Making use of the equation of state $p=\omega \rho$, a variation with respect to $a$ leads to

$c_{1}\left(2 \dot{H}+3 H^{2}\right)=V+\beta-\omega \rho$,

where

$c_{1}=(1-\alpha+\gamma)$.

Furthermore, the diffeomorphism invariance leads to the continuity equation

$\dot{\rho}+3 H(1+\omega) \rho=0$.

Finally, the equation of motion associated with $\phi$ is also present, but it is a consequence of the other equations of motion, thus it is trivially satisfied.

A remark is in order. The equation of motion (10) in this Hordenski mimetic model does not contain the Lagrange multipliers, and it is similar to the one valid in GR plus ordinary matter. However, here the mimetic potential $V$ appears in a very peculiar way, and this helps a lot in the search for exact solutions. In fact, one is dealing with a non-linear first order Riccati differential equation.

Furthermore, as in GR, one may have the de Sitter solution $H=H_{0}$ if and only if the potential is a constant, and $\omega=-1$ or $\rho=0$. Furthermore, one may have the de Sitter solution with vanishing potential and in the absence of matter, but with the constant $\beta \neq 0$. The effective cosmological constant depends on the ratio $\frac{\beta}{c_{1}}$. Thus, in the presence of a non-trivial potential, one may have only a quasi-de Sitter solution, and inflation and the current acceleration may be described. With regard to other solutions, there exist several possibilities.

\section{Absence of matter}

In the absence of matter, the Riccati equation may be recast in an homogeneous linear second order differential equation. In fact, introducing the new variable $y=a^{3 / 2}$, one has $H=\frac{2 \dot{y}}{3 y}$, and

$\ddot{y}-\frac{3}{4 c_{1}}(V(t)+\beta) y=0$.

The general solution of this kind of equation is not known. Approximate solutions may be investigated by the WKB method. Alternatively, one may use another approach, the so 
called adiabatic invariant method, and for the sake of completeness we report it in the Appendix. The reconstruction method is also possible and it has been investigated in [16]. In the following, we shall discuss some exact solutions.

As a first example, let us consider a quadratic potential,

$V=-\beta+2 V_{0}+\frac{3 V_{0}^{2}}{c_{1}}\left(\phi-\phi_{0}\right)^{2}$.

This choice gives

$V(t)=-\beta+2 V_{0}+\frac{3 V_{0}^{2}}{c_{1}}\left(t-t_{0}\right)^{2}$.

Then it is easy to show that the solution is

$y(t)=y_{0}^{\frac{3 V_{0}}{4 c_{1}}\left(t-t_{0}\right)^{2}}$,

and, in terms of the Hubble parameter, one has

$H(t)=\frac{V_{0}}{c_{1}}\left(t-t_{0}\right)$.

Since $H\left(t_{0}\right)=0$ and $\dot{H}\left(t_{0}\right)>0$, this is an example of a regular "bounce" solution. A solution of this kind for an extension of the Starobinski model has been found in [26], and for other bounce solutions, see [27].

Another bounce solution may be obtained by the following choice of $\beta=-2 c_{1} b^{2}$ and for the potential:

$V(\phi)=b^{2} c_{1} \frac{\sinh ^{2} b \phi}{\cosh ^{2} b \phi}$,

with $b$ a real parameter. In this case, the bounce solution is

$a(t)=a_{0} \cosh b t$,

with

$H(t)=b \frac{\sinh b t}{\cosh b t}$.

The bounce is at $t=0$. This kind of bounce solution may be obtained in a specific model of non-local gravity in FLRW space-time [28,29]. Here the bounce has been related to a simple potential in the mimetic scalar field.

Other exact solutions have been presented in [6].

\section{Presence of matter}

If matter is present, one may introduce the e-fold time $N=$ $\ln a$. As a result, the continuity equation becomes

$\frac{\mathrm{d} \rho}{\mathrm{d} N}=-3(1+\omega) \rho$

with solution

$\rho(N)=\rho_{0} \mathrm{e}^{-(1+\omega) N}$.
Furthermore, the equation of motion for $H$ becomes

$c_{1}\left(\frac{\mathrm{d} H^{2}}{\mathrm{~d} N}+3 H^{2}\right)=V+\beta-\omega \rho(N)$.

If

$c_{1}=(1+\gamma-\alpha) \neq 0$,

the solution is

$$
\begin{aligned}
& H^{2}(N) \\
& =\mathrm{e}^{-3 N}\left(C+\int \mathrm{d} N \mathrm{e}^{3 N} \frac{V(N)+\beta-\omega \rho_{0} \mathrm{e}^{-(1+\omega) N}}{c_{1}}\right),
\end{aligned}
$$

where $C$ is an integration constant. Furthermore, if $\omega+1$ is non-vanishing, one has

$$
\begin{aligned}
H^{2}(N)= & C \mathrm{e}^{-3 N}+\frac{\beta}{3 c_{1}}-\frac{\rho_{0} \omega}{\omega+1} \mathrm{e}^{-3 \omega N} \\
& +\int \mathrm{d} N \mathrm{e}^{3 N} \frac{V(N)}{c_{1}} .
\end{aligned}
$$

In the case $\omega=-1$, one instead has

$H^{2}(N)=C \mathrm{e}^{-3 N}+\frac{\beta}{3 c_{1}}+\frac{\rho_{0}}{c_{1}}+\mathrm{e}^{-3 N} \int \mathrm{d} N \mathrm{e}^{3 N} \frac{V(N)}{c_{1}}$.

Some comments are in order. Since

$t(N)=\int \frac{\mathrm{d} N}{H(N)}$

we may obtain $N=N(t)$ and $a(t)=\mathrm{e}^{N(t)}$. The contribution depending on $C$ is the contribution associated with the mimetic dark matter. The term depending on $\frac{\beta}{c_{1}}$ acts again as an effective cosmological constant. Another constant contribution may be obtained by the simplest choice for the potential $V=V_{0}$, namely a constant potential. In this case, we have

$H^{2}(N)=C \mathrm{e}^{-3 N}+\frac{\beta}{3 c_{1}}-\frac{\rho_{0}(1+\omega)}{\omega} \mathrm{e}^{-3(1+\omega) N}+\frac{V_{0}}{c_{1}}$

and

$H^{2}(N)=C \mathrm{e}^{-3 N}+\frac{\beta}{3 c_{1}}+\frac{\rho_{0} N}{c_{1}} \mathrm{e}^{-3 N}+\frac{V_{0}}{c_{1}}$.

For consistency, we have to assume $\beta+V_{0}>$. Thus, these solutions tend for large $N$ to the de Sitter space-time.

Another interesting example of the potential is

$V(N)=3 g(N)+\frac{\mathrm{d} g}{\mathrm{~d} N}$

with $g(N)$ a known function. In this case, the solution is

$$
H^{2}(N)=C \mathrm{e}^{-3 N}+\frac{\beta}{3 c_{1}}-\frac{\rho_{0}(1+\omega)}{\omega} \mathrm{e}^{-3(1+\omega) N}+\frac{V_{0}}{c_{1}} g(N) .
$$


As an example, take

$V(N)=V_{0}\left(N-N_{0}\right)^{b-1}\left(3\left(N-N_{0}\right)+b\right)$,

with $b \neq 2$. As a result

$$
\begin{aligned}
H^{2}(N)= & C \mathrm{e}^{-3 N}+\frac{\beta}{3 c_{1}}-\frac{\rho_{0}(1+\omega)}{\omega} \mathrm{e}^{-3(1+\omega) N} \\
& +\frac{V_{0}}{c_{1}}\left(N-N_{0}\right)^{b} .
\end{aligned}
$$

The nature of the solution may depend on the sign of $b$. To simplify the discussion, assume $C=\beta=\rho_{0}=0$, and $\frac{V_{0}}{c_{1}}>0$ Thus

$H(N)=\left(\frac{V_{0}}{c_{1}}\right)^{1 / 2}\left(N-N_{0}\right)^{b / 2}$.

As a consequence

$t-t_{0}=\left(\frac{V_{0}}{c_{1}}\right)^{-1 / 2} \frac{2}{2-b}\left(N-N_{0}\right)^{1-b / 2}$

and

$N-N_{0}=\left(\frac{V_{0}}{c_{1}}\right)^{1 /(2-b)}\left(\frac{2-b}{2}\left(t-t_{0}\right)\right)^{2 /(2-b)}$.

The Hubble parameter reads

$H(t)=A\left(t-t_{0}\right)^{b /(2-b)}$.

If $b>2$, as well as for $b<0$, there exists a future-time singularity (see, for example [30,31] and the references therein). If $0<b<2$, there is a bounce solution. For example, for $b=1$, one has the bounce solution

$H(t)=\frac{V_{0}}{c_{1}}\left(t-t_{0}\right)$.

In this case the potential is

$V(\phi)=V_{0}\left(1+\frac{3 V_{0}}{c_{1}}\left(\phi-\phi_{0}\right)^{2}\right)$,

in agreement with the result discussed in Sect. 3 .

\section{Conclusions}

In this paper, a specific cosmological Hordenski scalargravity mimetic model has been investigated within a FLWR space-time. The mimetic scalar field has been implemented making use of a Lagrange multiplier, and it has been shown that the model leads to equations of motion formally similar to the original simpler mimetic matter model of the so-called mimetic matter model [5]. Several exact solutions describing inflation, bounces, and future-time singularities have been presented and discussed.
It should be interesting to investigate a spherically symmetric static solution of this generalized mimetic model along the lines of Ref. [32].

Open Access This article is distributed under the terms of the Creative Commons Attribution 4.0 International License (http://creativecomm ons.org/licenses/by/4.0/), which permits unrestricted use, distribution, and reproduction in any medium, provided you give appropriate credit to the original author(s) and the source, provide a link to the Creative Commons license, and indicate if changes were made. Funded by SCOAP ${ }^{3}$.

\section{Appendix}

In the following we briefly discuss an alternative method to find the solution of a differential equation of the kind

$\ddot{y}+Q(t) y=0$.

The above linear homogeneous differential equation can be associated with the following non-linear differential equation, dubbed the Ermakov-Pinney equation (see [33-35]), namely:

$\ddot{u}+Q(t) u=\frac{h^{2}}{u^{3}}$.

In fact, the following result holds true; the solutions $y$ and $u$ are related by

$y=u \sin \theta, \quad \theta=\int \frac{h}{u^{2}} \mathrm{~d} t$,

and the constant $h$ is given by the so-called Lewis adiabatic invariant

$h^{2}=\frac{h^{2} y^{2}}{u^{2}}+(u \dot{y}-\dot{u} x)^{2}$.

As an example, let us consider

$Q(t)=\frac{q}{t^{4}}, \quad q>0$.

Then it is easy to show that an exact solution of the ErmakovPinney equation is

$u(t)=\left(\frac{h^{2}}{q}\right)^{1 / 4} t$

and

$\theta(t)=\theta_{0}-\frac{q^{1 / 2}}{t}$

As a result, the solution is

$y(t)=\left(\frac{h^{2}}{q}\right)^{1 / 4} t \sin \left(\theta_{0}-\frac{q^{1 / 2}}{t}\right)$, 
a non-trivial result. As a result, we may obtain an exact solution associated with a singular potential

$V(\phi) \equiv \phi^{-4}$.

Other exact solutions of the Ermakov-Pinney equation can be found in [33-35].

\section{References}

1. R. Adam et al. (Planck Collaboration), arXiv:1502.01582 [astroph.CO]

2. P. Ade et al. (Planck Collaboration), arXiv:1502.01589 [astroph.CO]

3. A.Y. Kamenshchik, E.O. Pozdeeva, A. Tronconi, G. Venturi, S.Y. Vernov, Class. Quantum Gravity 31, 105003 (2014). arXiv:1312.3540 [hep-th]

4. A.Y. Kamenshchik, E.O. Pozdeeva, A. Tronconi, G. Venturi, S.Y. Vernov, Class. Q. Grav. 33, 015004 (2016). arXiv:1509.00590 [gr$\mathrm{qc}]$

5. A.H. Chamseddine, V. Mukhanov, JHEP 1311, 135 (2013). arXiv:1308.5410 [astro-ph.CO]

6. A.H. Chamseddine, V. Mukhanov, A. Vikman, JCAP 1406, 017 (2014). arXiv:1403.3961 [astro-ph.CO]

7. L. Mirzagholi, A. Vikman, JCAP 1506(06), 028 (2015). arXiv: 1412.7136 [gr-qc]

8. N. Deruelle, J. Rua, JCAP 1409, 002 (2014). arXiv:1407.0825 [grqc]

9. A.O. Barvinsky, JCAP 1401(01), 014 (2014). arXiv:1311.3111 [hep-th]

10. J.D. Bekenstein, Phys. Rev. D 48, 3641 (1993). arXiv:gr-qc/9211017

11. F. Arroja, N. Bartolo, P. Karmakar, S. Matarrese, JCAP 1509, 051 (2015). arXiv:1506.08575 [gr-qc]

12. A. Golovnev, Phys. Lett. B 728, 39 (2014). arXiv:1310.2790 [grqc]

13. E.A. Lim, I. Sawicki, A. Vikman, JCAP 1005, 012 (2010). arXiv:1003.5751 [astro-ph.CO]
14. S. Capozziello, J. Matsumoto, S. Nojiri, S.D. Odintsov, Phys. Lett. B 693, 198 (2010). arXiv:1004.3691 [hep-th]

15. S. Nojiri, S.D. Odintsov, Mod. Phys. Lett. A 29(40), 1450211 (2014). arXiv:1408.3561 [hep-th]

16. J. Matsumoto, S.D. Odintsov, S.V. Sushkov, Phys. Rev. D 91(6), 064062 (2015). arXiv:1501.02149 [gr-qc]

17. S.D. Odintsov, V.K. Oikonomou, arXiv:1508.07488 [gr-qc]

18. R. Myrzakulov, L. Sebastiani, S. Vagnozzi, Eur. Phys. J. C 75, 444 (2015). arXiv:1504.07984 [gr-qc]

19. M. Raza, K. Myrzakulov, D. Momeni, R. Myrzakulov, arXiv:1508.00971 [gr-qc]

20. R. Myrzakulov, L. Sebastiani, S. Vagnozzi, S. Zerbini, Fund. J. Mod. Phys. 8, 119 (2015). arXiv:1505.03115 [gr-qc]

21. G.W. Horndeski, Int. J. Theor. Phys. 10, 363 (1974)

22. C. Deffayet, X. Gao, D.A. Steer, G. Zahariade, Phys. Rev. D 84, 064039 (2011). arXiv:1103.3260 [hep-th]

23. A. De Felice, T. Kobayashi, S. Tsujikawa, Phys. Lett. B 706, 123 (2011). arXiv:1108.4242 [gr-qc]

24. S. Nojiri, S.D. Odintsov, Phys. Lett. B 691, 60 (2010). arXiv:1004.3613 [hep-th]

25. G. Cognola, E. Elizalde, L. Sebastiani, S. Zerbini, Phys. Rev. D 83, 063003 (2011). arXiv:1007.4676 [hep-th]

26. R. Myrzakulov, L. Sebastiani, S. Zerbini, Eur. Phys. J. C 75(5), 215 (2015). arXiv:1502.04432 [gr-qc]

27. R. Myrzakulov, L. Sebastiani, Astrophys. Space Sci. 352, 281 (2014). arXiv:1403.0681 [gr-qc]

28. T. Biswas, T. Koivisto, A. Mazumdar, JCAP 1011, 008 (2010). arXiv: 1005.0590 [hep-th]

29. T. Biswas, A.S. Koshelev, A. Mazumdar, S.Y. Vernov, JCAP 1208, 024 (2012). arXiv:1206.6374 [astro-ph.CO]

30. S. Nojiri, S.D. Odintsov, S. Tsujikawa, Phys. Rev. D 71, 063004 (2005). arXiv:hep-th/0501025

31. G. Acquaviva, L. Bonetti, G. Cognola, S. Zerbini, Phys. Rev. D 88(12), 124024 (2013). arXiv:1309.6950 [gr-qc]

32. R. Myrzakulov, L. Sebastiani, Gen. Relativ. Gravit. 47(8), 89 (2015). arXiv:1503.04293 [gr-qc]

33. H.R. Lewis, J. Math. Phys. 9, 1976 (1968)

34. H.R. Lewis, W.B. Riesenfeld, J. Math. Phys. 10, 1458 (1969)

35. C.J. Eliezer, A. Gray, SIAM 30, 463 (1976) 